\title{
A Cognitive Analysis of the English Existential Construction from the Perspective of Iconicity
}

\author{
Luxi Yang \\ Modern English and Teacher Education Research Center, Chongqing Normal University, Chongqing, China \\ Email: ylx1024@gmail.com
}

\begin{abstract}
For the purpose of seeking the underlying motivations of the English existential construction, this paper first constructs the English representations of Idealized Cognitive Model construction pattern of existential construction and then, based on the theory of iconicity, attempts to explain the internal language structure from the external factors by exploring four kinds of mapping relationship between the linguistic structure of the English existential construction and the conceptual structure it represents, accordingly, to find out the cognitive motivations of the English existential construction forming. It can also make us grasp the nature of this language construction and better understand the construction forming motivations of this universal complex construction of almost all the human languages.
\end{abstract}

Index Terms - English existential construction, construction pattern, cognitive motivations, iconicity

Existential construction (henceforth EC) is a particular type of sentence structure appearing in almost all the languages in the world. Owing to its special linguistic characteristics, and its various representations in different languages, EC has attracted many scholars' attention. And they have made a deep research on it. However, cognitive motivations of existential construction forming have been paid little attention.

In recent two or three decades, with the development of language and cognition studies, people have found that there is a certain relationship between language structure and experiential structure. Cognitive linguistics claims that language is shaped or at least influenced by our experience of the world and the way we perceive and conceptualize it (Ungerer \& Schmid, 2001). The cognitive constraint and influence on language is first embodied by iconicity, Iconicity is a set of signs whose meaning in some crucial way resembles their form (Nanny \& Fischer, 1999:109). It denotes that linguistic structure is a reflection of cognitive or experiential structure. Iconicity is of great significance in revealing the mapping relationship between cognition and language structure.

As cognitive linguistics does not just concern about the real world of objects; but deals with the cognitive models we have about the world, it provides a new reference point for the iconic relation. It is the external factors such as reality; physiology, cognition, knowledge, meaning and pragmatics together that attribute to the motivation of linguistic form. The main principles of iconicity are manifestations of human's conceptualization processes.

This paper first attempts to put forward a universal Idealized Cognitive Mode (henceforth ICM) construction pattern of EC and to figure out the English representations of the ICM construction pattern of EC. And then it, based on the theory of iconicity, attempts to explain the internal language structure from the external factors by exploring four kinds of mapping relationship between the linguistic structure of the English EC and the conceptual structure it represents, accordingly, to find out the cognitive motivations of the English existential construction forming.

\section{THE ENGLISH REPRESENTATIONS OF THE ICM CONSTRUCTION PATTERN OF EXISTENTIAL CONSTRUCTION}

There are certain clusters of conditions that are more basic to human experience than other clusters and also more basic than individual conditions in the cluster. Lakoff and Johnson (1980) refer to such a cluster as an experiential gestalt, which is often representable by an Ideal Cognitive Model (Lakoff, 1987:345). An ICM can help better interpret and use its different manifestations.

And there are different representations of an ICM construction pattern in different languages, which result from different cognitive motivations. For the purpose of seeking the underlying motivations of the English EC, this paper first attempts to put forward a universal ICM construction pattern of EC and to figure out the English representations of the ICM construction pattern of EC (i.e. the English EC in this paper).

Existential construction refers to the construction that expresses the existence, appearance and disappearance of things, including human beings, animals, physical objects, or 'some place exists something'. All the existence involves existential space, existential way, and existential entity.

So existential construction needs three basic components: a locative phrase, a verb phrase and a noun phrase. The locative indicating existential space is placed before the noun phrase representing existential entity. The space -before-entity model has illustrated the relationship of existence and formed an image schema of 'in some place exists something'. The verb phrase denoting the existential ways is perceived on the basis of the space, therefore, is placed after the locative. After we have perceived the space and the ways of existence, the next to be perceived is the figure. Accordingly, the ICM construction patterns of the existential construction should be (Yang, 2007): 
a. Locative Phrase $+\mathrm{V}+\mathrm{NP}$.

b. There $+\mathrm{V}+\mathrm{NP}$. (Here, there is known as an introductory there.)

In English, the representations of the ICM construction pattern of EC are as the following: introductory word 'there'> existential way>existential entity > existential space (or existential time), or existential entity > existential space (or existential time), or existential entity. For example:

(1) a. A dog. (Existential entity)

b. A dog in the park. (Existential entity > existential space)

c. There lies a dog in the park. (Introductory word > existential way >existential entity >existential space)

If existential entity is omitted, the EC doesn't exist. For example:

(2) a.* In the park (only existential space)

b. ${ }^{*}$ Lies (only existential way)

c.* lies in the park (only existential space and existential way)

Obviously, a*, b*, c* can't be accepted.

Generally speaking, in EC, existential space/time is essential and necessary, but in English, it seems as if the existential space/time can be omitted. Actually, it is just because the introductory word "there" plays the part similar to space.

So the English representations of the ICM construction patterns of EC are(Yang,2007):

a. Locative Phrase + Existential V +Existential NP.

b. There + Existential V + Existential NP. (Here, there is known as an introductory there.)

\section{THE PRINCIPLES OF ICONICITY}

Iconicity is the similarity between the signifier and the signified. Language iconicity refers to the imitation phenomenon between the sound, form, and structure of language and the signified. Theory of language iconicity was put forward in contrast to language arbitrariness and has been developing gradually.

Iconicity is important cognitive linguistic theory, which is the study of the similarity or natural relationship between the linguistic structure and the conceptual structure it represents. Therefore, it can help reveal the underlying cognitive mechanisms which motivate the linguistic manifestations of the English EC.

Motivation refers to the fact that language structures directly reflect the structures of reality or the conceptual structures. The main principles of motivation in the current research are: the principle of sequence iconicity, the principle of quality iconicity, the principle of distance iconicity and principle of markedness iconicity. Literature on iconicity seems to emphasize motivation, i.e. how linguistic structures reflect aspects of the structure of reality or aspects of human conceptualization of reality.

\section{A. The Principle of Sequence Iconicity}

According to Givon, the principle of sequence iconicity is that "the order of the element in language parallels that in physical experience or the order of knowledge" (1985: 103).

We can probe the principle of order iconicity from three perspectives:

1. Iconicity in the Basic Sentence Order

2. The Semantic Principle of Linear Order

3. The pragmatic Principle of Linear Order

The use of this principle is reflected in the clause-initial placement of indefinite or important full-NP referents in languages with flexible word order (Givon 1988). It can also be reflected in the clause-initial placement of contrastive topics, cleft-focused topics, L-dislocated topics and WH-questioned constituents.

Cognitively, less predictable information is likely to be more urgent than predictable, accessible information. And both, 'surprising' and 'important' are identified as the less frequent, marked figure which needs attention, thus tend to be placed first in the string.

\section{B. The Principle of Quantity Iconicity}

The quantity of linguistic expressions may correspond to the quantity, importance and predictability of concepts. John Haiman (1983) puts forward the term of the iconicity of length: "the length of an utterance may correspond to the extent to which it conveys new or unfamiliar information." Jakobson (1965) uses the notion of "complexity iconicity" to discuss the length of linguistic signs and the complexity degree of the concepts they convey. Haiman (1985) believes "Formal complexity corresponds to conceptual complexity." Givon (1994: 49) puts forward the quantity iconicity by saying that "One finds the footprints of this principle all over the syntactic map, in the assignment of larger segmental size or more prominent intonation to information that is semantically larger, less predictable, or more important". He states this principle as:

(i) A large chunk of information will be given a larger chunk of codes.

(ii) Less predictable information will be given more coding material.

(iii) More important information will be given more coding material.

In fact the cognitive basis of this principle lies in the fact that the increasing amount of linguistic material will draw 
more of people's attention, which leads to complexity in mental processing so as to convey more information.

\section{The Principle of Distance Iconicity}

The principle of distance iconicity is also known as the principle of proximity iconicity or proximity principle. Givon adopted the term of "proximity principle" and provided us with such argument as "The closer together two concepts are semantically or functionally, the more likely they are to be put adjacent to each other lexically, morphotactically or syntactically". (Givon, 1985)

\section{The Principle of Markedness Iconicity}

The notion of 'markedness' entered structural linguistics via Prague School initially as a term in language analysis. According to Wang Yin, the sequence of syntactic constituents from the unmarked to the marked in syntactic constructions corresponds to the natural sequence of information arrangement in our conceptual structures, and the marked constituents indicate additional information.

The unmarked term refers to the element with common meanings and usages. The marked term refers to the element with distinctive features in one pair. Compared with the marked term, the unmarked term usually has more generalized and even neutral meaning. It is predictive in communication and has wider scope of distribution. Frequently, we ask: "How long is the stick?" but not "How short is the stick?" The unmarked term long, wide, high, deep, etc., are neutral in expressing length, width, height, depth, etc. Yet their corresponding marked terms only refer to the concepts of shortness, narrowness, lowness, shallowness, etc. The unmarked-marked dichotomy can be applied to almost every level of language: phonological, morphological and syntactic.

\section{An ANALYsis of THE ENGLISH EXISTENTIAL CONSTRUCTION From THE PERSPECTIVE OF ICONICITY}

\section{A. Sequence Iconicity of the English Existential Construction}

The principle of temporal sequence is the most natural sequence, which is called PTS (Principle of Temporal Sequence) by Tai (Haiman, 1985b). He defines it as: "the relative word order between two syntactic units is determined by the temporal order of the states which they represent in the conceptual world" (ibid, 1985b). So the temporal sequence of "V+ NP" denotes the short mental perception process of the action of existence, appearance or disappearance of an entity. And the action is always perceived first, then the agent. What's more, in such short perception process, the agent/the existential entity is impossible immediately to be perceived clearly and specifically, therefore it is indefinite and new to the perceiver.

Besides the temporal sequence, sequence iconicity in temporal scope and spatial scope is also an important principle.

According to Tai (ibid, 1985b), some word order phenomena involving time expressions in Chinese can be explained better in terms of the notion of temporal scope rather than temporal sequence. Scope implies a special sequential relation. The principle of temporal scope (PTSC) can be stated as:

If the conceptual state represented by a syntactic unit $\mathrm{X}$ fall within the temporal scope of the conceptual state represented by a syntactic unit $\mathrm{Y}$, then the word order is YX. PTSC requires a constituent with smaller temporal range should follow one with larger range (Haiman, 1985b: 60).

Consider a Chinese example:

(3) Zuotian wo he xiaoli qu le shudian.

Yesterday, I went to the bookshop with xiaoli.

The temporal range of the actions denoted by the verb in the above Chinese example are within the temporal range denoted by the time adverbial in bold, the verb are thus put after the time adverbial.

In cognition, temporal category and spatial category are considered as two basic categories and they are interrelated. Spatial category is based on our bodily experience and then it maps its concept onto the temporal category. As there is the principle of temporal scope, this principle can also be possible in spatial category, that is, concepts with a larger scope precede those with a smaller scope. For example:

(4) zai chufang 1i de zhuozi de shangmian de hezi 1i you yibeng shu.

There is a book in the box on the top of the table in the kitchen.

The principle of temporal scope reflects the word order from the whole to the part or from the ground to the figure. Thus the relationship between the ground and the figure is as follows:

(i) The ground provides the existing space for the figure: The ground is a frame embodying the figure and the figure is the object the ground embodies.

(ii) The shape of the ground is larger, stationary, not clear and shapeless; on the contrary, the figure is smaller, mobile, salient and clear.

Though Tai's PTSC reflects mostly the conceptualization processes of Chinese nation, the form of the Chinese EC is actually the same with the locative subject EC in English, we may assume that two nations have the similar conceptualization processes in forming EC and can apply Tai's principle to the analysis of the English EC.

According to the viewpoint of figure-ground segregation, people can perceive the figure from the spatial perspective or perceive space from the perspective of the figure. Here consider the former perspective. And first consider the locative subject EC: 
(5) On the top of the mountain lived a hunter.

(5) Is a typical EC according to Freeze's study of the EC in the world for the locative is at the beginning. In terms of Tai's principle, ' a hunter ' is clearly within the spatial scope of ' On the top of the mountain ', so the locative indicating a ground is placed before the noun phrase representing a figure. The ground-before-figure model has illustrated the relationship of existence and formed an image schema of 'in some place exists/appears/disappears something'. As for the existential ways denoted by the verbs of 'existence', 'appearance' or 'disappearance', they are perceived on the basis of the ground, therefore, are placed after the ground. After we have perceived the ground and the ways of existence, the next to be perceived is the figure. Actually, this model also is accordance with the ICM construction patterns of EC.

Next let's consider the preformed EC:

(6) There appear some clouds in the sky.

(7) There is a God.

(6) And (7) are both the introductory there EC. They are different from the locative subject EC only in that the ground is abstract. However, it is very clear that the figures 'some clouds ' and 'a God' are within the spatial scope of the abstract location denoted by there.

But why are there two forms of the English EC and different grounds? Actually, when uttering (5), the speaker is just an onlooker, he can vividly perceive the scene of existence, and introduce it to the hearer. But when uttering (6) and (7), the speaker has just an image schema of existence in the mind, and the process of uttering is the sequence that the speaker presents to the hearer: first an abstract location realized by there, and then the action which happens in the location, and finally the entity. Though these processes all happen in the mind, they are the reflection of the reality in practical.

And why is the introductory there EC more natural and preferred to express the concept of existence?

In the introductory there EC, there provides a general abstract ground, and uttering "there + be/VI" seems to be opening a container in order to let us see the content in it, and implies the coming of the information of something existence, it also provides the hearer with a mental preparation for the coming information. According to the ICM pattern of existential construction, existential ways are always paid little attention. So the hearer will pay more attention to the following new information: the existential entity and the specific existential space. However, how should the information be arranged? Based on our cognitive experience, the information order will be affected by the following factors:

a .Human`s language expression habits: first important, then less important.

b. The natural sequence of syntax: the figure-and-ground model.

c. The ICM pattern of existential construction: existential entity>existential space (refer to importance).

d. The two principles of information coding:

(1) Entity argument > setting argument;

(2) Definite argument > indefinite argument.

Therefore, the information order should be: existential entity + existential space. i.e. the word order should be: NP + Locative Phrase (sometimes omitted just because it is the shared given information). Obviously, the introductory there EC can better reflect human`s cognitive processes of the concept of "someplace exists something". This is why it is preferred to locative subject EC.

Up to now, we can see that the ground is before the figure in both the introductory there EC and the locative subject ES regardless the ground is abstract or specific. Therefore, the linguistic sequence of the English EC reflects in some way the conceptual sequence in perceiving "someplace exists something".

\section{B. Quantity Iconicity of the English Existential Construction}

We know the principle of quantity iconicity is that more linguistic signs employed suggest more conceptual meaning conveyed. Newmeyer (1998) proposes that the greater the syntactic or morphological complexity, the more elements capable of bearing meaning; the more elements of meaning, the more complex the meaning (158-159). Here let's make an illustration of quantity iconicity of the English ES. First consider the following examples:

(8) There exists a God.

(9) There occurred a sudden revolution in public taste.

First, let's give an interpretation of the occurrence of the specific location. In terms of Givon's principle (b) of quantity iconicity_- "Less predictable information will be given more coding material", the specific location of the figure in (9) is less predictable than that in (8) in that it gives more linguistic signs to the location. When uttering (8), the speaker assumes that the hearer can figure out that location on the basis of shared given information. But when uttering (9), the speaker assumes that the location of the figure is not clear to the hearer, and not easy to think of, so the speaker has to provide sufficient information for the hearer to make out the location of the figure. In terms of Givon's principle (c) of quantity iconicity_-"More important information will be given more coding material", since the location in (9) contains more coding material, the location "in public taste" is thus important or surprising information because we normally assume the revolution happens in some place instead of "in public taste", which is really beyond our expectations. In (8), the speaker makes no mention of the specific location, which indicates the specific location of the figure is not important information and not necessary to give. 
Second, let's talk about the length of the verb phrases and the noun phrases. The noun phrases are much longer than the verb phrases in (8) and (9), which can be explained by the quantity principle and indicates that the noun phrases are less predictable and carry more important information than the verb phrases. As we all know, the attention of the English EC is gradually shifted from the ground to the figure, which is what the speaker wants to convey to the hearer, and is also the function of the English EC, and thus the noun phrases convey more new content than the verb phrases. The following sentences verify that the verb phrase cannot be much longer (or have much more informational content) than the noun phrase: (Lakoff, 1987: 571)

(10) a. *Suddenly there burst into the room a cop.

b. $*$ Suddenly there burst into the room a tall cop.

c.?*Suddenly there burst, into the room a brawny cop.

d.?*Suddenly-there burst into the room a mean-looking cop.

e. Suddenly there burst into the room a tall, brawny, mean-looking cop.

In the opinion of Lakoff, those sentences get progressively better with the addition of elements to the noun phrases. He also argues that the verb phrase is just to "set up an appropriate background against which the noun phrase will count as new information" (Lakoff, 1987: 571).

In the introductory there EC, the occurrence of a specific location is a manifestation that the specific location is unpredictable and also needs the hearer`s attention, and the omission of a specific location indicates that the specific location is predictable or unimportant or unnecessary to occur. These examples of the English EC just demonstrate that linguistic complexity is a reflection of conceptual complexity. A common form of iconicity is the expression of relatively simple ideas or situations in short, simple sentences, and of complex ideas or situations in complex sentences. In brief, more linguistic signs imply more meanings, and fewer linguistic signs, fewer meanings, which constitutes the basic tenets of quantity iconicity.

\section{Distance Iconicity of the English Existential Construction}

Haiman (1983: 782) expounds the first iconic motivation — "the linguistic distance between expressions corresponds to the conceptual distance between them". Here are the other two iconic motivations which result in the distance between expressions:

(i) The linguistic separateness of an expression corresponds to the conceptual independence of the object or event which it represents.

(ii) The social distance between interlocutors corresponds to the length of the message, referential content being equal.

In Haiman's opinion, if an element incorporated with another element forms a constituent, the two elements are predicted to have a close semantic relationship; if two elements are separated, the relationship is thus not close. By studying expressions like 'lighthouse keeper' and 'light housekeeper', Bolinger (1977) found that the contrast between the two expressions was not one of relative stress, but of the ratio of linguistic distance, measured in time units between the morphemes light, house, keeper. Their conclusion was that:

Since in lighthouse keeper the semantic bond between light and house is closer than that between house and keeper (immediate constituents are lighthouse keeper), and since the disjunctives transparently (iconically) supply a physical separation whose width correlates inversely with the semantic bond, it follows that the disjunctives function directly to carry the information" (Bolinger, 1977).

However, not only distance but closeness, belongs to the proximity principle. Haiman (1985b: 228) points out what belongs together mentally is placed close together syntactically.

In this section we will find out the iconic motivations through investigating the distance or closeness of linguistic expressions and the conceptual distance or closeness they denote in the English EC. First let's consider the following sentences:

(11) a. There entered a policeman in the room.

b. There came into the room a policeman.

We have presented in section 3.2 that the occurrence of the specific location indicates the unpredictability and importance of that location, and that the noun phrase is also what the speaker wants to convey to the hearer. Therefore, two image schemata unfold before us in (11a): the first image is that a policeman entered a certain space, and another one is that the policeman is in the room, which is the image the speaker wants to convey to the hearer. However, the first image schema evoked in (11b) is a moving path and the destination formed by "come into the room", and then an image of "a policeman" is evoked, which alone composes the new information. In terms of our analysis, the noun phrases and the locative phrase in (11a) are close in that they form a concrete image of existence. The locative phrase and the noun phrase in (11b) are separated; accordingly their conceptual distance is comparatively large and the specific location of (11b) is more independent of noun phrases than that in (11a). (11b) seems to emphasize the action process by "come into the room".

In the English EC, the locative phrase and the noun phrase attach to the verb closely, that is to say, the conceptual distance is close.

The setting, the action and the participant in the EC are three basic constituents, which denote respectively the three construction components: the existential space, the existential way and the existential entity. The existential space is the 
base of existence, and the ground on which the existential way happens, so the conceptual distance between the sitting and the action are close. While the existential entity is the agent of the existential way, certainly the conceptual distance between the entity and the action are also close. Therefore, conceptually the sitting and the entity are inseparable with the action, and thus the distance between them is close. The action should be put between the sitting and the participant, and connects the two constituents. What's more, the existential space is the ground, the existential entity the figure, only against the ground can the figure single out; accordingly the conceptual distance of the two is close. So the figure and the ground can appear closely together in the introductory there EC. All the conceptual distances can then be reflected on the linguistic representation, that's to say, the two forms of EC:

(1) Locative Phrase $+\mathrm{V}+\mathrm{NP}$

(2) There + V + NP (+Locative Phrase)

\section{Markedness Iconicity of the English Existential Construction}

According to Wang Yin, the sequence of syntactic constituents from the unmarked to the marked in syntactic constructions corresponds to the natural sequence of information arrangement in our conceptual structures, and the marked constituents indicate additional information.

The ordering from the unmarked to the marked is in accordance with the natural cognitive ordering. John Haiman (1980a: 528) argued that "It is universally assumed that markedness is iconically motivated: categories that are marked morphologically and syntactically are also marked semantically."

In Wang`s (1999) opinion, natural sequence, that is, the subject is before the transitive verb, is the unmarked because it tallies with the regular cognitive sequence of "the agent>action". But if the sentence is inverted and the agent is put after the verb, the sentence is the marked. Consider the following example:

(12) a. Our teacher sat in the front seat.

b. In the front seat sat our teacher.

Compared with (12a) in natural sequence, the inverted construction of (12b) is marked and carries extra meaning, drawing our attention to what happened to the front seat, that is, the less predictable person: our teacher.

Traditionally, the English EC is considered as an inverted construction with the subject after the verb; accordingly, it may be regarded as the marked since the natural sequence is the unmarked.

a. Predicate Locative (Theme+V+Locative):

(13) The book is on the table.

b. EC (Locative+V+Theme):

(14) a. On the table is a book.

b. There is a book on the table.

Sentence (13) is a typical unmarked sequence with a theme subject and the constituents are arranged according to the natural word order of English language. But (14) is the marked with a locative subject because we have verified that there is meaningful and supposed that it refers to an abstract space, a mental place. Since the marked indicates extra information, it draws our attention to what happens to the locative. That's to say, what happens to the locative is important and less predictable, so it needs our more attention. Accordingly, NP becomes a salient item in the marked form.

In selecting the word order, people tend to put the perceptually salient item or the more attractive item in the first place. Therefore, in the unmarked form the subject, which is definite and the figure within the profiled relationship, is more prominent than the locative; however, in the marked form the locative subject more prominent than the noun phrase is selected as the conceptual starting point. Meanwhile, it functions to evoke new information.

\section{SUMMARY}

This paper first constructs the ICM representations of the English EC and then gives a general interpretation of the English EC by virtue of the four principles of iconicity. The principle of sequence iconicity analyzed the scope sequence of the English EC: from the ground to the figure. The principle of quantity iconicity gave an explanation for the occurrence of the specific location in the introductory there EC and the relative length between the noun phrases and the verb phrases. The principle of markedness iconicity illustrated the markedness of the English EC. The principle of proximity iconicity expounded the independence of the specific location, the closeness between the participant and the verb, and the closeness between the setting and the verb, the closeness between the noun phrase and the locative phrases.

The research finds that the sequence of the English EC tallies with the sequence of the information transfer, and with the sequence of the ICM construction patterns of EC and information-coding; the distance between the components of the English EC is in accordance with the distance of their corresponding concepts; in the introductory there EC, the occurrence of the specific location is related to the predictability of its concept, and it indicates the importance or unpredictability of the noun phrase that the length of the noun phrase should be equal to or longer than the length of the verb phrase; and the ground-before-figure model of the English EC is contrary to the figure-ground model of natural word order, which reflects that the ground, as the conceptual starting point of the English EC, is the focus of cognition, and thus is prominent. 


\section{REFERENCES}

[1] Bolinger, D. (1977). The Form of Language. London: Longman.

[2] Givon, T. (1985). Iconicity, Isomorphism and Non-Arbitrary Coding in Syntax. In J. Haiman(ed.), Iconicity in Syntax. Amsterdam: John Benjamins, 187-220.

[3] Givon, T. (1988). Mind, Code and Context. Hillsadale, NJ: Academic Press.

[4] Givon, T. (1994). Isomorphism in the Grammatical Code: Cognitive and Biological Considerations. In M. Simone (ed.), Iconicity in Language. Amsterdam: John Benjamins.

[5] Haiman, J. (1980). The Iconicity of Grammar: Isomorphism and Motivation. Language, 56, 515-540.

[6] Haiman, J. (1983). Iconic and Economic Motivation. Language, 59, 24-32.

[7] Haiman, J. (1985a). Iconicity in Syntax. Amsterdam, Philadelphia: John Benjamins.

[8] Haiman, J. (1985b). Natural Syntax. Cambridge: CUP.

[9] Haiman, J. (1985c). Iconicity in Syntax TSL 6. Amsterdam: John Benjamins.

[10] Jacobson, R. (1965). Quest for the Essence of Language. Diogenes, 45, $23-31$.

[11] Lakoff, G. (1987). Women, Fire and Dangerous Things. Chicago: University of Chicago Press.

[12] Lakoff, G. \& M. Johnson. (1980). Metaphors we live by. Chicago: University of Chicago Press.

[13] Nanny, M. \& G. Fisher. (1999). Form Meaning. Amsterdam: John Benjamins Publishing Co.

[14] Newmeyer, F.J. (1998). Language Form and Language Function. Cambridge: The MIT Press.

[15] Ungerer, F. H. \& J. Schmid. (2001). An Introduction to Cognitive Linguistics. London: Addison Welsley Longman Limited.

[16] Yan,Wu. (2006). A Study of English Existential Sentence from the Perspective of Iconicity. Master dissertation, Changsha University.

[17] Luxi, Yang. (2007). A Cognitive Approach to the English Existential Construction. Master dissertation, Chongqing University.

[18] Yang, Gu. (1997). The Existential Sentences in Chinese. Modern Foreign Languages, 3.

[19] Wenli, Du. (1996). A Discussion on Language Iconicity. Journal of Sichuan International Studies University, 1.

[20] Yin, Wang. (1999). On the Iconicity of the Language Signs. Beijing: Xinhua Press.

Luxi Yang was born in Sichuan, China in 1965. She received her M.A. degree in linguistics and Applied Linguistics from Chongqing University, China in 2007.

She is currently an associate professor in the School of Foreign Languages and Literature, Chongqing Normal University, Chongqing, China. Her research interests include psycholinguistics and cognitive linguistics.

Prof. Yang is a member of the Chongqing Cognitive linguistics Association. 